\title{
Elderly women used interdisciplinary approaches to face obstacles and make a successful transition through recovery after hip fracture
}

Robinson SB. Transitions in the lives of elderly women who have sustained hip fractures. J Adv Nurs 1999 Dec;30:1341-8.

QUESTION: What are elderly women's experiences of the factors that promote function and enable a successful transition through the recovery process after a hip fracture?

Design

Grounded theory.

Setting

Midwestern USA.

\section{Participants}

15 English speaking women $>70$ years of age (age range $72-82$ y, $100 \%$ white) who were recovering from hip fracture, had been discharged from a subacute unit in the previous 9 months, had returned home to live alone, and were cognitively intact. All women had children who visited at least weekly.

\section{Methods}

Focus groups (3 groups of 7, 5, and 3 women, respectively) were held in a small, comfortable room within a 250 bed hospital. Predetermined, open ended questions were used to encourage participants to talk openly about the transitional conditions through recovery from hip fracture. During the sessions, non-verbal behaviour was recorded and important points were validated by the moderator. Sessions were audiotaped and analysed using open coding, a concept map, and axial coding.

\section{Main findings}

2 main categories were identified. The first category, function inhibiting factors, described the obstacles to successful transition after hip fracture. These factors were physical discomfort, such as fatigue and chronic pain; feeling limited, which was expressed as dependency, loss of control, impatience, and fear of falling; bending precautions needed for proper healing of the hip, which affected social and home maintenance activities; need for devices to assist them, which were viewed as hindrances and visible stigmas that reflected a loss of independence; and loss of enabling skills, such as stair climbing and driving. Women used 6 adaptive methods to cope with their limited functional ability: viewing age as strength, looking ahead, confronting problems head on with determination and perseverance, minimising the significance of problems, seeing humour in frustration, and having faith. The second category, function promoting factors, described the approaches that the women took to enhance their ability to make a successful transition after hip fracture. These approaches were recognising progress, which was confirmed by self and other's assessment and helped to overcome feelings of depression; making adaptations for daily activities by using creativity and by learning from caregivers; and accepting help from family, friends, and the community to compensate for shortcomings. Women expressed a sense of well being, thankfulness, and pride from having experienced successful hip fracture recovery.

\section{Conclusions}

Elderly women faced obstacles to successful transition after hip fracture but found adaptive approaches to enhance their inner strength and promote their recovery. These women used a variety of interdisciplinary interventions to enable a successful recovery and restore a sense of personal well being.

\section{COMMENTARY}

The study by Robinson adds to our understanding of older women's lived experience after traumatic hip injury by shifting attention from physiological outcome measures to factors that promote recovery. The mortality $(14 \%-36 \%$ after $1 \mathrm{y}$ ) and morbidity associated with fractured hips in older adults are indications of the importance of this research topic. ${ }^{1}$

Story telling in focus groups is a good data collection method for qualitative studies because it gives insight into the language used and the meaning of events held by the participants. The study was based on the lived experience of 15 women, and therefore, the findings may differ from other populations. Furthermore, participants were described as cognitively intact but it is unclear how cognition was assessed.

The risk of dependency after hip fracture is high. Understanding how to protect functional independence and community competency are essential to nursing practice. This study links interdisciplinary interventions to promoting recovery. As such, the clinical relevance of this study for subacute units is high because the results give direction for potential interventions for nurses. Clinical utility could be strengthened with a clearer description of the multidimensional components of functional ability. The author writes "Through the qualitative approach, the definition of functional status can be expanded to encompass psychological components" (p 1346). This is in keeping with gerontological nursing assessment where functional status is understood to include psychological components. Other researchers have also shown that functional ability involves physical, psychological, and social functioning. ${ }^{2}$ ?

This study offers nurses in subacute units direction for providing specific interventions targeted toward holistic recovery that goes beyond bone healing and reaches into elements of quality of life.

Belinda Parke, RN, MSN Clinical Nurse Specialist Victoria General Hospital Capital Health Region Victoria, British Columbia, Canada

1 Kannus P, Parkkari L, Sievanen H, et al. Epidemiology of hip fractures. Bone 1996;18(suppl 1):57S-63S.

2 Stone JT, Wyman JF, Salisbury SA, et al. Clinical gerontological nursing: a guide to advanced practice. 2nd edition. Philadelphia: WB Saunders Company, 1999

3 Kane RA, Kane RL. Assessing the elderly: a practical guide to measurement. Lexington, MA: Lexington Books, 1981. 